\title{
Note on Separability of the Werner states in arbitrary dimensions *
}

\author{
Arthur O. Pittenger $\dagger^{\ddagger}$ and Morton H. Rubin $\ddagger$ \\ $\dagger$ Department of Mathematics and Statistics, University of Maryland, Baltimore County, Baltimore, MD 21228-5398 \\ $\ddagger$ Department of Physics, University of Maryland, Baltimore County, Baltimore, MD 21228-5398
}

(February 22, 2000)

\begin{abstract}
Great progress has been made recently in establishing conditions for separability of a particular class of Werner densities on the tensor product space of $n d$-level systems (qudits). In this brief note we complete the process of establishing necessary and sufficient conditions for separability of these Werner densities by proving the sufficient condition for general $n$ and $d$.
\end{abstract}

03.67.Lx, 03.67.Hk, 03.65.Ca

We consider the $d^{n}$-dimensional Hilbert space

$$
H^{\left[d^{n}\right]}=H^{[d]} \otimes \cdots \otimes H^{[d]}
$$

composed of the direct product of $n d$-dimensional Hilbert spaces. As in [1] we let $\tilde{j}$ denote the $n$-tuple $j \ldots j$ and define $|\tilde{j}\rangle=|j\rangle \otimes \cdots \otimes|j\rangle$. The particular class of generalized Werner state, $W^{\left[d^{n}\right]}(s)$, considered here is defined as the convex combination of the completely random state $\frac{1}{d^{n}} I^{(n)}$ and an entangled pure state $|\Psi\rangle\langle\Psi|$,

$$
W^{\left[d^{n}\right]}(s)=(1-s) \frac{1}{d^{n}} I^{(n)}+s|\Psi\rangle\langle\Psi|
$$

where $I^{(n)}$ is the identity operator on the $d^{n}$-dimensional Hilbert space. To be specific, take

$$
|\Psi\rangle=\frac{1}{\sqrt{d}} \sum_{j=0}^{d-1}|\tilde{j}\rangle .
$$

The Werner state was originally defined in 2 for two qubits. Its generalization has been applied to study Bell's inequalities and local reality, and it has served as a test case of separability arguments in a number of studies. The problem treated here is to determine necessary and sufficient conditions on the parameter $s$ so that $W^{\left[d^{n}\right]}(s)$ is fully separable. That is

$$
W^{\left[d^{n}\right]}(s)=\sum_{a} p(a) \rho^{(1)}(a) \otimes \cdots \otimes \rho^{(n)}(a)
$$

where the $\rho^{(r)}(a)$ are density matrices on the respective $d$-dimensional Hilbert spaces and the set of numbers $\{p(a)\}$ is a probablility distributuion. For references to many of the related studies and for the relevance of the Werner states to the study of entanglement the reader can consult [1] 3 - 5 .

As shown in [4], a necessary condition for separability for all $d$ and $n$ follows from the Peres partial transpose condition [6] or from a weaker condition that can be proved via the Cauchy-Schwarz inequality. Specifically suppose $j=j_{1} \ldots j_{n}$ and $k=k_{1} \ldots k_{n}$ differ in each component: $j_{r} \neq k_{r}$. Let $u$ and $v$ be indices with $u_{r} \neq v_{r}$ and $\left\{u_{r}, v_{r}\right\}=$ $\left\{j_{r}, k_{r}\right\}$. Then for fully separable states $\rho$

$$
\left(\sqrt{\rho_{j, j}} \sqrt{\rho_{k, k}}\right) \geq\left|\rho_{u, v}\right|,
$$

where $\rho$ is written as a matrix in the computational basis defined by the tensor products of $\left|j_{i}\right\rangle\left\langle k_{i}\right|, 1 \leq i \leq n$. Choosing $j$ and $k$ appropriately in (3), we obtain the necessary condition

$$
s \leq\left(1+d^{n-1}\right)^{-1},
$$

and special cases of this condition were derived in [5, ],8], for example.

\footnotetext{
${ }^{*}$ This work was supported in part by the National Security Agency.

${ }^{\ddagger}$ Present address: The Centre for Quantum Computation, Clarendon Laboratory, Oxford University
} 
The remaining challenge has been to show that this necessary condition is also sufficient, and various partial results have been obtained in the papers just cited. In particular, (4) was shown in [3] to be sufficient for all $d$ and $n=2$ and in [1] sufficiency was established for $d$ prime and all $n$. In this note we complete the study of this aspect of the Werner states by proving the sufficiency part of the following result.

Theorem: The Werner density $W^{\left[d^{n}\right]}(s)$ is fully separable if and only if $s \leq\left(1+d^{n-1}\right)^{-1}$.

The relevant technique combines a representation of fully separable states presented in 3 with an induction argument presented in [1]. Let $s=\left(1+d^{n-1}\right)^{-1}$. Then it is easy to show that

$$
W^{\left[d^{n}\right]}(s)=\frac{1}{1+d^{n-1}}\left(\frac{1}{d} \sum_{j=0}^{d-1}|\tilde{j}\rangle\langle\tilde{j}|\right)+\frac{d^{n-1}}{1+d^{n-1}}\left(\frac{1}{d^{n}}\left(I^{(n)}+\sum_{j \neq k}|\tilde{j}\rangle\langle\tilde{k}|\right)\right) .
$$

Since the first term in (5) is a sum of separable projections, the proof reduces to showing the separability of the second term. It is convenient in what follows to intorduce a set of fixed phases and to show that

$$
\rho^{(n)}=\frac{1}{d^{n}}\left(I^{(n)}+\sum_{j \neq k} \zeta_{j}|\tilde{j}\rangle\langle\tilde{k}| \zeta_{k}^{*}\right)
$$

where $\left\{\left|\zeta_{r}\right|=1, r=0, \cdots, d-1\right\}$ is separable.

We proceed by induction. When $n=1$, (6) becomes

$$
\rho^{(1)}=\frac{1}{d}\left(\sum_{j=0}^{d-1} \sum_{k=0}^{d-1} \zeta_{j}|j\rangle\langle k| \zeta_{k}^{*}\right)
$$

which is obviously a projection for any choice of the parameters $\zeta_{r}$. Now assume that the density matrix of the form in (6) is fully separable for $n$; then we shall show that it is fully separable for $n+1$. Following the ideas in [3], for a fixed choice of parameters $\zeta_{r}$ define

$$
\mathbf{w}^{(m)}=\left(\zeta_{0} z_{0}^{(m)}, \cdots, \zeta_{d-1} z_{d-1}^{(m)}\right)
$$

where $z_{j}^{(m)} \in\{ \pm 1, \pm i\}$ and $\zeta_{r}$ is independent of $m$. We have a total of $4^{d}$ different vectors. It is easy to check that if we sum over all $m$,

$$
\sum_{m} \mathbf{w}_{r}^{(m)}=\zeta_{r} \sum_{m} z_{r}^{(m)}=0, \quad \sum_{m}\left(\mathbf{w}_{r}^{(m)}\right)^{2}=\zeta_{r}^{2} \sum_{m}\left(z_{r}^{(m)}\right)^{2}=0, \text { and } \quad \sum_{m}\left|\mathbf{w}_{r}^{(m)}\right|^{2}=4^{d} .
$$

For each $\mathbf{w}^{(m)}$ define the product state $\rho(m)=\rho^{(n)}\left(\mathbf{w}^{(m)}\right) \otimes \rho^{(1)}\left(\mathbf{z}^{(m) *}\right)$ where $\mathbf{z}^{(m)}$ is equal to $\mathbf{w}^{(m)}$ with all the $\zeta_{r}$ 's equal to 1 and

$$
\begin{aligned}
\rho^{(n)}\left(\mathbf{w}^{(m)}\right) & =\frac{1}{d^{n}}\left(I^{(n)}+\sum_{j \neq k} \zeta_{j} z_{j}^{(m)}|\tilde{j}\rangle\langle\tilde{k}| \zeta_{k}^{*} z_{k}^{(m) *}\right) \\
\rho^{(1)}\left(\mathbf{z}^{(m)}\right) & =\frac{1}{d}\left(I^{(1)}+\sum_{r \neq s} z_{r}^{(m)}|r\rangle\langle s| z_{s}^{(m) *}\right) .
\end{aligned}
$$

The state $\rho(m)$ is separable by the induction hypothesis,and it follows that the convex combination $\rho^{(n+1)}=$ $\frac{1}{4^{d}} \sum_{m} \rho(m)$ is also separable. Now we multiply out the terms:

$$
\begin{aligned}
\rho^{(n+1)} & =\frac{1}{d^{n+1}}\left(I^{(n+1)}+I^{(n)} \otimes A^{(1)}+A^{(n)} \otimes I^{(1)}+B\right) \\
A^{(1)} & =\sum_{r \neq s}|r\rangle\langle s| \frac{1}{4^{d}} \sum_{m} z_{r}^{(m) *} z_{s}^{(m)} \\
A^{(n)} & =\sum_{j \neq k} \zeta_{j} \zeta_{k}^{*}|\tilde{j}\rangle\langle\tilde{k}| \frac{1}{4^{d}} \sum_{m} z_{j}^{(m)} z_{k}^{(m) *} \\
B & =\sum_{j \neq k} \sum_{r \neq s} \zeta_{j} \zeta_{k}^{*}|\tilde{j}\rangle\langle\tilde{k}|\otimes| r\rangle\langle s| \frac{1}{4^{d}} \sum_{m} z_{j}^{(m)} z_{k}^{m *} z_{r}^{(m) *} z_{s}^{(m)} .
\end{aligned}
$$


Since the components of $\mathbf{w}^{(m)}$ are chosen independently of one another, $\sum_{m} z_{r}^{(m) *} z_{s}^{(m)}=0$ for $r \neq s$; consequently, $A^{(n)}$ and $A^{(1)}$ vanish. As noted in [3] the choice of the $\mathbf{w}^{(m)}$ also simplifies the remaining term, since for $r \neq s$ and $j \neq k$

$$
\frac{1}{4^{d}} \sum_{m} z_{j}^{(m)} z_{k}^{(m) *} z_{r}^{(m) *} z_{s}^{(m)}=\delta(j, r) \delta(k, s)
$$

where $\delta(r, s)$ is the Kronecker delta. Then

$$
\rho^{(n+1)}=\frac{1}{d^{n+1}}\left(I^{(n+1)}+\sum_{j \neq k} \zeta_{j}|\tilde{j} j\rangle\langle\tilde{k} k| \zeta_{k}^{*}\right),
$$

which is of the same form as (6) with $n \rightarrow n+1$, completing the induction step and the proof of the theorem. .

[1] A. O. Pittenger and M. H. Rubin, "Separability and Fourier representations of density matrices", quant-ph/0001014, submitted for publication to Phys. Rev. A.

[2] R. F. Werner, Phys. Rev. A 40, 4277 (1989).

[3] P. Rungta, W. J. Munro, K. Nemoto, P. Deuar, G. J. Milburn, and C. M. Caves, "Qudit Entanglement", quant-ph/0001075.

[4] A. O. Pittenger and M. H. Rubin, "Complete separability and Fourier representations of n-qubit states", quant-ph/9912116, submitted for publication to Phys. Rev. A.

[5] S. L. Braunstein, et. al., Phys. Rev. Lett. 83, 1054 (1999).

[6] A. Peres, Phys. Rev. Lett. 77, 1413 (1996).

[7] C. M. Caves, G. J. Milburn, "Qutrit entanglement," quant-ph/9910001 (1999)

[8] W. Dür, J. I. Cirac, and R. Tarrach, Phys. Rev. Lett. 83, 3562 (1999). 